EPJ Web of Conferences 70, 00050 (2014)

DOI: $10.1051 /$ epjconf/ 20147000050

(C) Owned by the authors, published by EDP Sciences, 2014

\title{
New Physics Searches with ATLAS
}

\author{
Alaettin Serhan Mete ${ }^{\mathrm{a}}$ \\ ${ }^{1}$ On behalf of the ATLAS Collaboration \\ Department of Physics and Astronomy, University of California, Irvine, CA 92697
}

\begin{abstract}
Highlights from new physics searches with the ATLAS detector at the CERN Large Hadron Collider are presented. Results are based on the analysis of data collected in $p p$ collisions at a center-of-mass energy of $7 \mathrm{TeV}$ corresponding to integrated luminosities of 1-5 $\mathrm{fb}^{-1}$. No excess beyond the Standard Model expectations is observed.
\end{abstract}

\section{Introduction}

The high energy collisions at the CERN Large Hadron Collider (LHC) provide new opportunities to search for physics beyond the Standard Model (SM) of strong and electroweak interactions. This document highlights some of the searches performed using $7 \mathrm{TeV} p p$ collision data collected with the ATLAS detector [1] during 2011 and corresponding to total integrated luminosities that vary from 1 to $5 \mathrm{fb}^{-1}$.

\section{Search for high-mass di-lepton resonances}

Existence of heavy neutral gauge bosons, commonly denoted as $Z^{\prime}$, are predicted by many extensions to the SM [2-4]. In general, these hypothetical particles are most easily searched for in the leptonic decay channels due to relatively low and well understood SM background. The ATLAS search [5] summarized below is performed in the muon and electron channels. Results are also combined to further extend the reach.

In the electron channel events are sampled using a di-electron trigger that requires at least two electromagnetic clusters in the calorimeter, each with a transverse energy $\left(E_{\mathrm{T}}\right)$ of at least $20 \mathrm{GeV}$. Sampled events are further required to have two electron candidates with $E_{\mathrm{T}}>25 \mathrm{GeV}$ and $|\eta|<$ 2.47; the transition region between the barrel and endcap calorimeters, namely $1.37 \leq|\eta| \leq 1.52$, is excluded. Only the so-called medium electrons [6] that pass a set of stringent quality cuts are considered. To suppress background from photon conversions both electrons are required to have a hit in the first active pixel layer, whereas only the higher $E_{\mathrm{T}}$ electron is required to be isolated by requiring $\sum E_{\mathrm{T}}(\Delta R<0.2)<7 \mathrm{GeV}$, where $\Delta R=\sqrt{(\Delta \phi)^{2}+(\Delta \eta)^{2}}$ and $\sum E_{\mathrm{T}}(\Delta R<0.2)$ is the sum of the transverse energy deposition in the calorimeter around the electron direction, to suppress the QCD multi-jet background while maintaining a high signal selection efficiency.

In the muon channel events are sampled using a single muon trigger that requires the presence of a muon with a transverse momentum $\left(p_{\mathrm{T}}\right)$ of at least $22 \mathrm{GeV}$. Sampled events are further required to

a e-mail: amete@cern.ch

This is an Open Access article distributed under the terms of the Creative Commons Attribution License 2.0, which permits unrestricted use, distribution, and reproduction in any medium, provided the original work is properly cited. 
have two muon candidates with opposite charge and $p_{\mathrm{T}}>25 \mathrm{GeV}$. Those muons that are considered in this analysis are reconstructed independently both in the inner detector (ID) and the muon spectrometer (MS). To ensure a good momentum resolution, muons are required to pass a set of stringent quality cuts in these respective detectors. To suppress background from cosmic rays, the z position of the primary vertex is required to be within $200 \mathrm{~mm}$ of the center of the detector and the muon tracks are required to have a transverse impact parameter $\left|d_{0}\right|<0.2 \mathrm{~mm}$ as well as $\left|z_{0}\right|<1 \mathrm{~mm}$. To reduce background from jets, each muon is also required to be isolated such that the $p_{\mathrm{T}}$ sum of other tracks with $p_{\mathrm{T}}>1 \mathrm{GeV}$ around the direction of the muon, within a cone of $\Delta R=0.3$, is less than $5 \%$ of the $p_{\mathrm{T}}$ of the muon.

In both channels, those events that pass the afore mentioned selection criteria are kept for the final analysis. For each event the invariant mass of the di-lepton system $\left(m_{\ell \ell}\right)$ is constructed and an excess above the total background is searched for in the region $m_{\ell \ell}>70 \mathrm{GeV}$. The $m_{\ell \ell}$ distributions in both the electron and the muon channels are shown in Figure 1 for both data and the total expected background including the systematic uncertainties. The dominant contribution to the total background comes from the high mass tail of the SM $Z / \gamma^{*}$ production. No excess above the SM expectation is observed in either channel. Therefore, limits are set in production cross section times branching ratio, $\sigma B$, as a function of the hypothesis mass, $m_{Z^{\prime}}$. To fully exploit the search potential, results from each channel are statistically combined and the final result is shown in Figure 2. A sequential SM $Z^{\prime}$ is excluded at $95 \% \mathrm{CL}$ for masses up to $2.21 \mathrm{TeV}$. Limits are also set for other beyond SM signatures. More information can be found in [5].
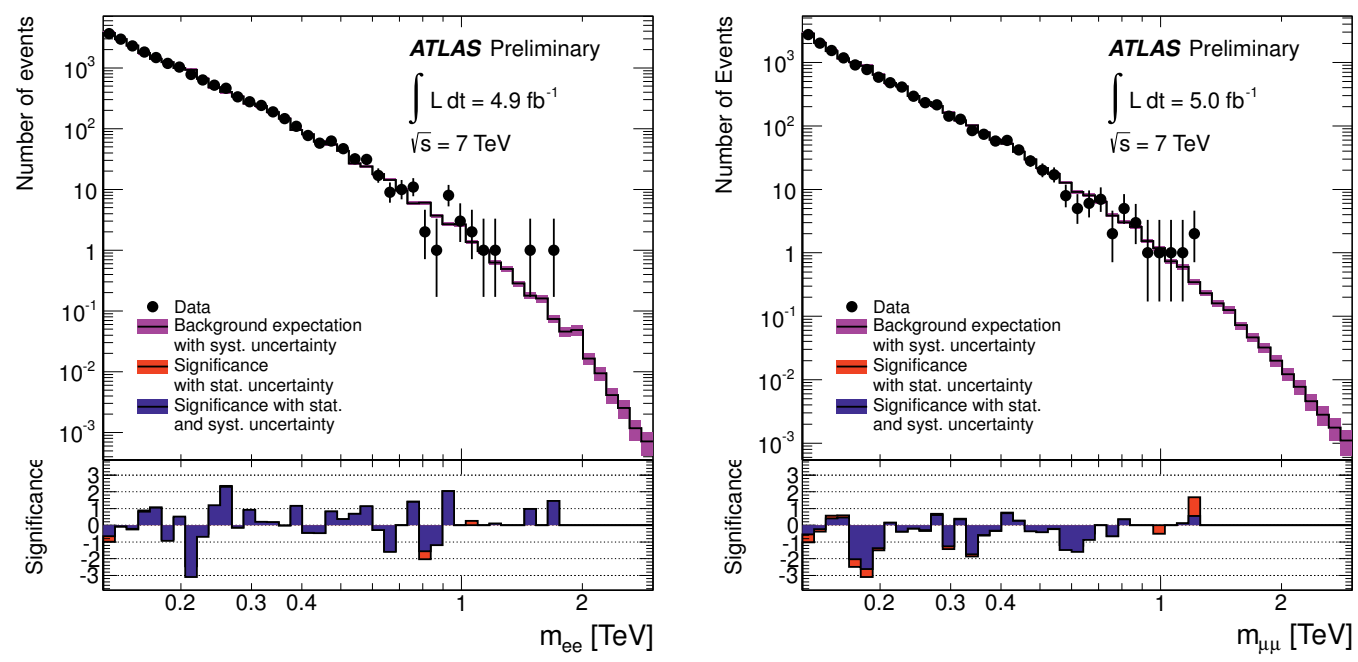

Figure 1. The di-electron (left) and the di-muon (right) invariant mass distributions after final selection compared to the expected background [5]. The bottom parts show the significance of the difference between data and expectation in each bin.

\section{Search for high-mass di-jet resonances}

With the $7 \mathrm{TeV} p p$ collisions, CERN's LHC reaches the highest collision energy ever reached at a collider. Large momentum transfer in these collisions results in high jet multiplicities with large 


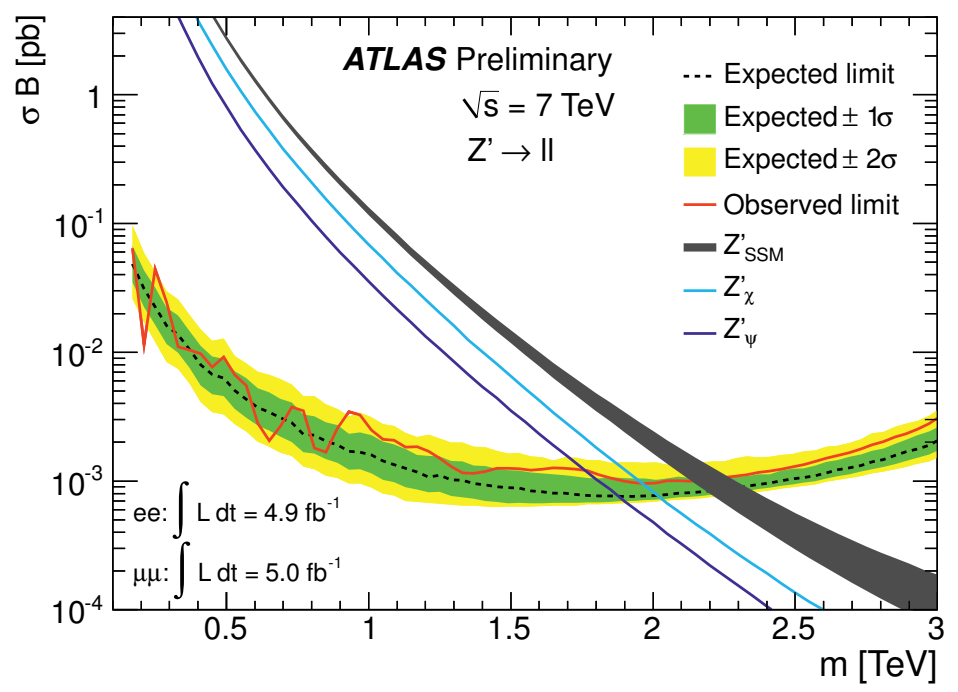

Figure 2. The expected and observed $95 \%$ C.L. upper limits, as well as the $68 \%$ and $95 \%$ contours of the expected limits, on $\sigma B$ as a function of mass for SSM $Z^{\prime}$ of the combination of electron and muon channels [5].

transverse momenta that gives an invaluable opportunity to test the SM and look for new physics [7, 8]. The ATLAS detector is used to search for new phenomena in di-jet mass and angular distributions [9]. Here the emphasis will be given to the search for excited quarks, $q^{*}$, in the di-jet mass spectrum of this comprehensive analysis.

A single jet trigger that requires a large transverse energy deposition in the calorimeter is used for this analysis. Events are required to pass an extensive set of selection criteria to ensure high quality and have at least two high $p_{\mathrm{T}}$ jets. Both the leading (i.e. highest $p_{\mathrm{T}}$ - denoted by subscript 1 from now on) and next-to-leading (denoted by subscript 2) jets are required to have in-time energy depositions to ensure their association with the $p p$ collision. In addition, among all the selected jets, there must be no poorly measured one with $p_{\mathrm{T}}$ greater than $30 \%$ of the $p_{\mathrm{T}}$ of the next-to-leading jet. In order to obtain a sample enriched in the hard scattering part of the phase space, the events must further satisfy $\left|y^{*}= \pm \frac{1}{2}\left(y_{1}-y_{2}\right)\right|<0.6$ and $\left|y_{1,2}\right|<2.8$. Here $y^{*}$ are the rapidities of the two highest $p_{\mathrm{T}}$ jets in their $\mathrm{CM}$ frame and $y_{1,2}$ are their rapidities in the $p p$ system with rapidity defined as $y=\frac{1}{2} \ln \left(\frac{E+p_{z}}{E-p_{z}}\right)$ where $E$ is the energy of the particle and $p_{z}$ is its momentum along the beam axis.

Those events that pass the afore mentioned selection criteria are kept for the final analysis. For each event the invariant mass of the di-jet system $\left(m_{j j}\right)$ is constructed using the two highest $p_{\mathrm{T}}$ jets and an excess above the background is searched for in the region $m_{j j}>850 \mathrm{GeV}$. The total background is obtained directly from the data where a fit to the $m_{j j}$ distribution is performed in a control region, which is then extrapolated to the signal region accounting for the systematic uncertainties that arise due to this procedure. The resulting $m_{j j}$ spectrum is shown in Figure 3. As can be seen, no excess above the background is observed. Therefore, limits are set on cross section times acceptance, $\sigma \times \mathcal{A}$, as a function of particle mass $m_{j j}$, which is also shown on Figure 3. With these results, excited quarks are excluded at $95 \%$ CL for masses up to $3.35 \mathrm{TeV}^{1}$. 

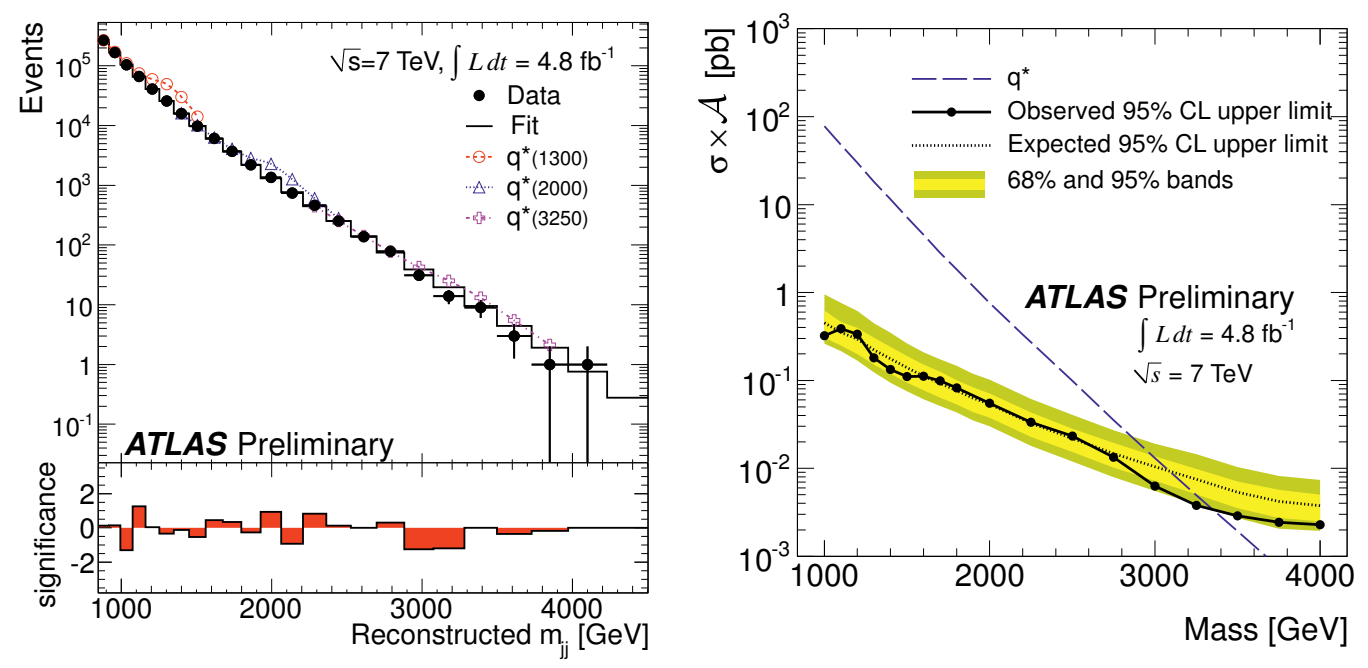

Figure 3. Left plot shows the di-jet invariant mass distribution after final selection compared to the expected background [9]. The bottom part of the same plot shows the significance of the difference between data and expectation in each bin. Right plot shows the the expected and observed 95\% C.L. upper limits, as well as the $68 \%$ and $95 \%$ contours of the expected limits, on cross section times acceptance, $\sigma \times \mathcal{A}$, as a function of particle mass $m_{j j}[9]$.

\section{Search for resonant $W Z$ production}

Existence of heavy charged gauge bosons, commonly denoted as $W^{\prime}$, are predicted by many extensions to the SM $[1,1,1]$. The ATLAS search [1] for such a particle decaying to $W Z \rightarrow \ell v \ell^{\prime} \ell^{\prime}$ $\left(\ell, \ell^{\prime}=e, \mu\right)$ final states is summarized in this section with the emphasis given to the Extended Gauge Model (EGM) $W^{\prime}$ of [1].

In this analysis four decay channels evee, ev $\mu \mu$, $\mu v e e$ and $\mu \nu \mu \mu$ are analyzed separately and then combined. Events are sampled with single electron or muon triggers with thresholds of $E_{\mathrm{T}}>20 \mathrm{GeV}$ and $p_{\mathrm{T}}>18 \mathrm{GeV}$, respectively.

Electrons are required have $E_{\mathrm{T}}>25 \mathrm{GeV}$ and $|\eta|<2.47$ excluding the transition region between the barrel and endcap calorimeters that was defined earlier. As in the high-mass di-lepton resonances analysis, only electrons that satisfy the requirements of the medium criteria are considered. To ensure electrons are originating from the primary vertex of the event, impact parameter cuts are also applied: $\left|d_{0}\right|<10 \sigma_{d_{0}}$ and $\left|z_{0}\right|<10 \mathrm{~mm}$. To select the prompt electrons from $W / Z$ decays, an isolation cut is also applied by requiring the sum of the $E_{\mathrm{T}}$ of the clusters around the electron within a cone of $\Delta R=0.3$ to be less then $4 \mathrm{GeV}$.

Muons are required to have $p_{\mathrm{T}}>25 \mathrm{GeV}$ and $|\eta|<2.4$ with tracks reconstructed both in the ID and the MS independently and pass a set of quality cuts in the respective sub-detectors to ensure a good quality. To suppress the multi-jet background, muons are required to be isolated such that the $p_{\mathrm{T}}$ sum of other tracks with $p_{\mathrm{T}}>1 \mathrm{GeV}$ around the direction of the muon, within a cone of $\Delta R=0.2$,

\footnotetext{
${ }^{1}$ At the time of writing this document, a more recent study is released by the ATLAS Collaboration that uses $p p$ collisions at a center-of-mass energy $\sqrt{s}=8 \mathrm{TeV}$ corresponding to an integrated luminosity of $5.8 \mathrm{fb}^{-1}$ that further extends the limit on excited quarks up to $3.66 \mathrm{TeV}[1]$.
} 
is less than $10 \%$ of the $p_{\mathrm{T}}$ of the muon. In conjuction to the electrons, muons are also required to be compatible with the primary vertex by requiring $\left|d_{0}\right|<10 \sigma_{d_{0}}$ and $\left|z_{0}\right|<10 \mathrm{~mm}$.

Based on the object selection described above, events that have an opposite charge same flavor lepton pair with an invariant mass within $20 \mathrm{GeV}$ of the $Z$ boson mass, a third lepton and a missing transverse energy of at least $25 \mathrm{GeV}$ are kept. The transverse mass of the reconstructed $W$ boson, defined as $m_{\mathrm{T}}^{W}=\sqrt{2 p_{\mathrm{T}}^{\ell} E_{\mathrm{T}}^{\text {miss }}(1-\cos \Delta \phi)}$ where $p_{\mathrm{T}}^{\ell}$ is the transverse momentum of the third lepton and $\Delta \phi$ is the opening angle between this lepton and $E_{\mathrm{T}}^{\mathrm{miss}}$ on the transverse plane, is constructed and required to be greater than $15 \mathrm{GeV}$ to suppress multi-jet background. Events satisfying all these selection criteria are kept for the final analysis. For these events, the transverse mass of the $W Z$ system, $m_{\mathrm{T}}^{W Z}=\sqrt{\left(E_{\mathrm{T}}^{Z}+E_{\mathrm{T}}^{W}\right)^{2}-\left(p_{x}^{Z}+p_{x}^{W}\right)^{2}-\left(p_{y}^{Z}+p_{y}^{W}\right)^{2}}$ where $E_{\mathrm{T}}^{Z}$ and $E_{\mathrm{T}}^{W}$ are the scalar sums of the transverse energies of the decay products of the $Z$ and $W$ candidates, respectively, is calculated and used to search for new physics. The $E_{\mathrm{T}}^{\text {miss }}$ vector is used as the estimator of the transverse momentum of the neutrino arising from the $W$ boson decay. The resulting distribution for the combination of all channels is shown in Figure 4. No excess above the SM expectation is observed; hence limits are set on the production cross section times branching ratio, $\sigma \times B$, as a function of $W^{\prime}$ mass. The limit plot is also shown in the same figure. As a result, an EGM $W^{\prime}$ is excluded at 95\% CL for masses up to 760 $\mathrm{GeV}$.
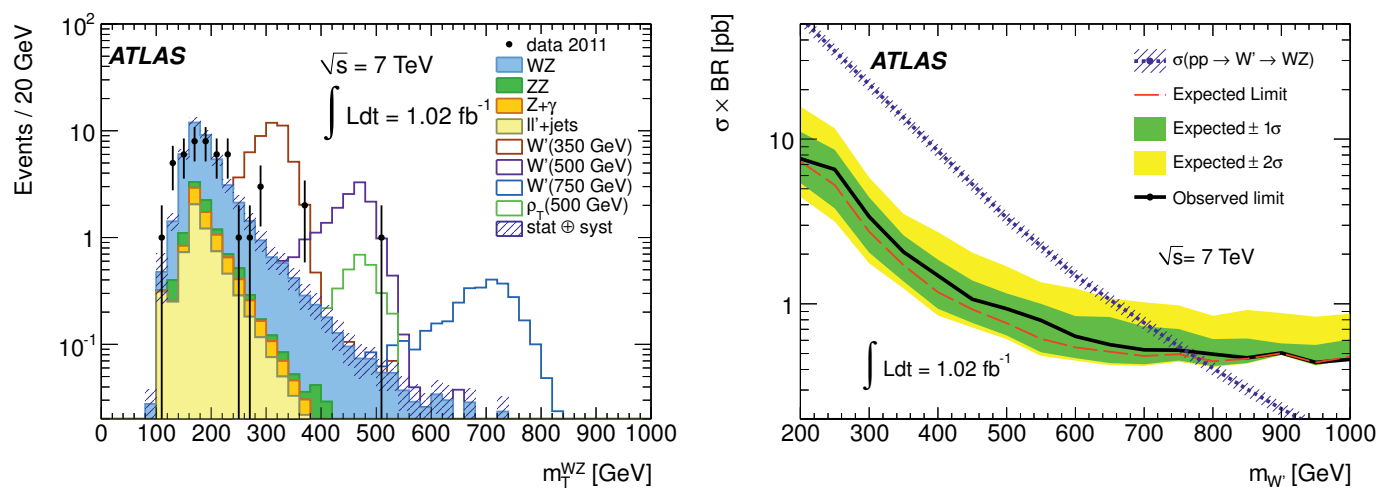

Figure 4. Left plot shows the observed and predicted $m_{\mathrm{T}}^{W Z}$ distribution for events with all selection cuts applied [1]. Predictions from different hypothetical signal samples are also shown. The right plot shows the observed and expected limits on $\sigma \times B$ as a function of $m_{W^{\prime}}$ [1]. The theoretical prediction is also shown taking into account various sources of systematic uncertainties.

\section{Search for heavy neutrinos and $W_{R}$ bosons}

Another important new physics signature that can be probed at the LHC energies is the production of heavy neutrinos. The ATLAS search [1] concentrates on two main production mechanisms: Lagrangian of effective operators [1] and Left-Right Symmetric Models (LRSM) [1, 1, 1]. This document is focused on the latter, within which the electroweak part of the SM is extended by a new gauge group giving rise to new force carriers; right-handed charged heavy gauge bosons, $W_{R}$, and neutral heavy gauge boson, $Z^{\prime}$. The decay chain that is considered in this search is: $q \bar{q} \rightarrow W_{R} \rightarrow \ell N$, where the heavy neutrino (either Majorana or Dirac type), $N$, decays via $N \rightarrow \ell W_{R}^{*} \rightarrow \ell j j$, resulting in 


\section{EPJ Web of Conferences}

a final state with two prompt leptons (either electron or muon), and two jets. Both the scenarios of no-mixing and maximal mixing between two generations of lepton flavors are investigated assuming that the mass difference between the heavy neutrinos is negligible. The object and event selection is as follows.

Electrons are required to pass the medium criteria, have $E_{\mathrm{T}}>25 \mathrm{GeV}$ and $|\eta|<2.47$ excluding the transition region between the barrel and endcap calorimeters. They are required to have a hit in the first active pixel layer, if expected, to suppress background from photon conversions. To suppress non-prompt background, each electron is required to be isolated such that the $\sum E_{\mathrm{T}}(\Delta R<0.3)$ is less than $15 \%$ of the $E_{\mathrm{T}}$ of the electron. Additionally, an electron whose track matches the ID track of a muon candidate is rejected.

Muons are required to have $p_{\mathrm{T}}>25 \mathrm{GeV}$ and $|\eta|<2.4$ with tracks reconstructed both in the ID and the MS independently and pass a set of quality cuts in the respective sub-detectors to ensure a good quality. To suppress non-prompt background, each muon is also required to be isolated as the electrons, using a slightly more sophisticated logic that is powerful in rejecting background muons and highly efficient for selecting signal muons produced in the decays of heavy neutrinos and reconstructed near the signal jets in cases where the heavy neutrino is boosted. Muons are also required to be compatible with the primary vertex by requiring $\left|d_{0}\right|<0.2 \mathrm{~mm},\left|d_{0}\right|<5 \sigma_{d_{0}}$ and $\left|z_{0}\right|<5 \mathrm{~mm}$.

Jets are reconstructed using the anti- $k_{\mathrm{t}}$ algorithm $[2,2]$ with a radius parameter of $R=0.4$. Only those that have $p_{\mathrm{T}}>20 \mathrm{GeV}$ and $|\eta|<2.8$ are considered. Jets that are closer to an electron than $\Delta R=0.5$ are not used to avoid possible double counting. To enrich the sample with jets originating from the hard scattering, at least $75 \%$ of the summed $p_{\mathrm{T}}$ of all reconstructed tracks associated with a jet with $|\eta|<2.8$ is required to come from tracks originating from the selected primary vertex. Additional quality cuts to suppress possible detector background are also applied as explained in [1].

According to the object selection defined above, events are preselected by requiring exactly two leptons (either an electron or a muon) and at least one jet. To suppress the SM $Z / \gamma^{*}$ production, the invariant mass of the di-lepton system, $m_{\ell \ell}$, is required to be greater than $110 \mathrm{GeV}$. Events are then grouped into two signal regions as: Same Sign (SS) and Opposite Sign (OS) di-lepton events. To further suppress the background in the OS di-lepton channel, the scalar sum of the transverse energies of the two leptons and the leading two jets with $p_{\mathrm{T}}>20 \mathrm{GeV}$, denoted by $S_{T}$, is required to be greater than $400 \mathrm{GeV}$. The mass of the heavy neutrino, $N$, can be reconstructed from its decay products of one lepton and two jets. In those cases where $N$ has a large momentum, i.e. is boosted, the hadronic decay products can be reconstructed as a single jet due to their proximity to each other, which accounts for up to $50 \%$ of the signal events where the mass splitting between $W_{R}$ and $N$ is large. Therefore, the invariant mass of the $W_{R}$ boson, $m_{l l j(j)}$, is reconstructed from the leptons and the two highest $p_{\mathrm{T}}$ jets in events with at least two jets, or a single jet in events with only one jet. This variable is required to be greater than $400 \mathrm{GeV}$, and is used as the final discriminant to search for new physics in the LRSM. The final $m_{l l j(j)}$ distributions for the data, the total predicted background and two selected hypothetical signal points are shown in Figure 5 for both the OS and SS signal regions. The uncertainties on the total background predictions are also shown in both cases. The most dominant background in the OS signal region is the SM $Z+$ jets production, whereas in the SS signal region is the so-called "fake lepton(s)", which arises from SM $W+$ jets, $t \bar{t}$, and multi-jet production where one or more jets are misidentified as prompt isolated leptons and estimated using a "data-driven" method.

The Dirac type heavy neutrinos are only searched for in the events with OS di-lepton pairs as their signature don't allow production of same sign lepton pairs. However, the Majorana type heavy neutrinos are searched for in both signal regions. No excess above the SM expectation is observed in either signal region. Figure 6 shows the exclusion limits for the masses of heavy neutrinos and the $W_{R}$ boson in the LRSM interpretation, both for the no-mixing and maximal-mixing scenarios between $N_{e}$ 
and $N_{\mu}$ neutrinos, for both the Majorana and Dirac heavy neutrinos hypotheses. For both no-mixing and maximal-mixing scenarios, $W_{R}$ bosons with masses up to $\approx 1.8 \mathrm{TeV}(\approx 2.3 \mathrm{TeV})$ are excluded for mass differences between the $W_{R}$ and $N$ masses larger than $0.3 \mathrm{TeV}(0.9 \mathrm{TeV})$.
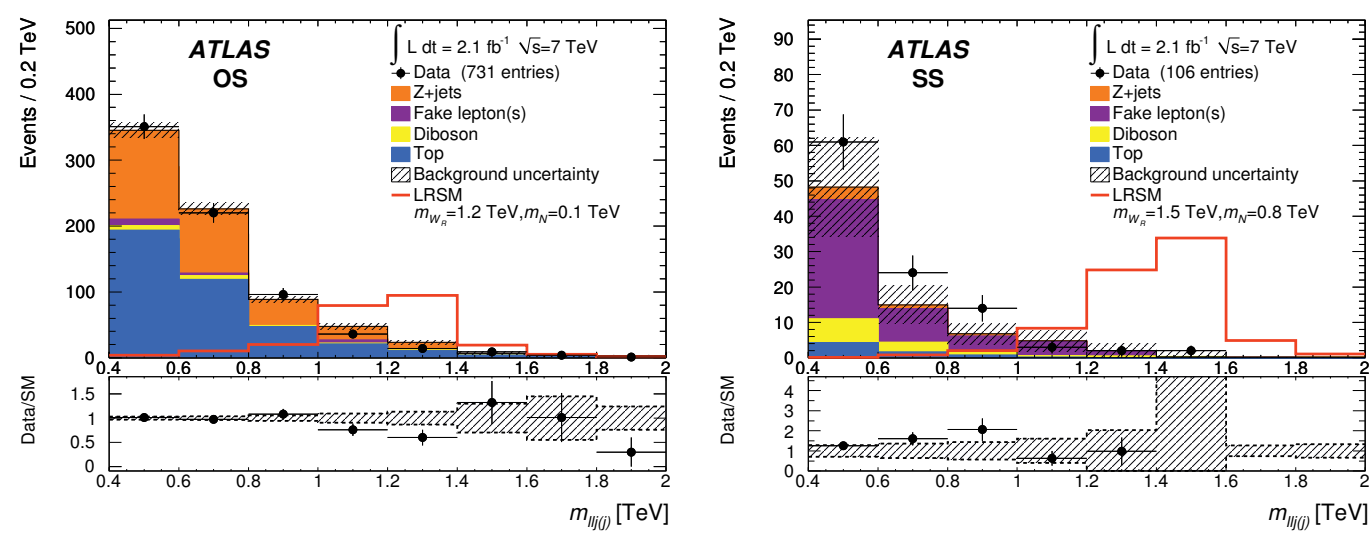

Figure 5. Left plot shows the distributions of the reconstructed $W_{R}$ invariant mass, $m_{l l j(j)}$, for the OS and the right one for the SS di-lepton events [1]. The hypothetical signal distributions for $m_{W_{R}}=1.2 \mathrm{TeV}$ and $m_{N}=0.1 \mathrm{TeV}$, and $m_{W_{R}}=1.5 \mathrm{TeV}$ and $m_{N}=0.8 \mathrm{TeV}$ are shown, respectively.
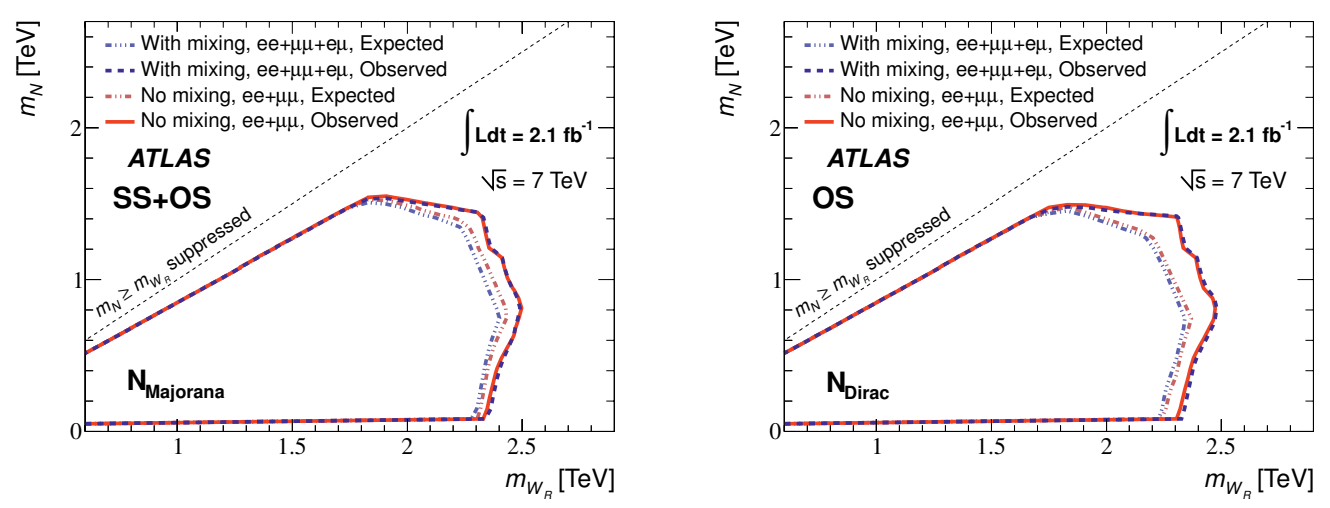

Figure 6. Expected and observed 95\% C.L. upper limits on the heavy neutrino and $W_{R}$ masses for the Majorana case (left) and Dirac case (right), in the no-mixing and maximal-mixing scenarios [1].

\section{Conclusions}

Some of the most recent new physics searches, at the time of the conference, with the ATLAS detector at the CERN LHC are highlighted. Those analyses that are mentioned in this document search for new physics signatures other then supersymmetry (SUSY). Results are based on the analysis of data 
collected in $p p$ collisions at a center-of-mass energy of $7 \mathrm{TeV}$ corresponding to varying integrated luminosities between 1-5 $\mathrm{fb}^{-1}$ depending on the particular study. No excess beyond the Standard Model expectations is observed in any of the analysis. Hence, limits are set on various observables. These limits include, but not limited to, $m_{Z^{\prime}}$ up to $2.21 \mathrm{TeV}$ and $m_{q^{*}}$ up to $3.35 \mathrm{TeV}$ at $95 \% \mathrm{CL}$. Figure 7 summarizes the mass reach of a representative selection of ATLAS searches for new phenomena other than SUSY. Data corresponding to approximately $30 \mathrm{fb}^{-1}$ of $p p$ collisions at a center-of-mass energy of $8 \mathrm{TeV}$ are planned to be collected during the full 2012 data taking period. This new data will be even more fruitful for testing the SM and searching for new physics.

\section{ATLAS Exotics Searches* - 95\% CL Lower Limits (Status: March 2012)}

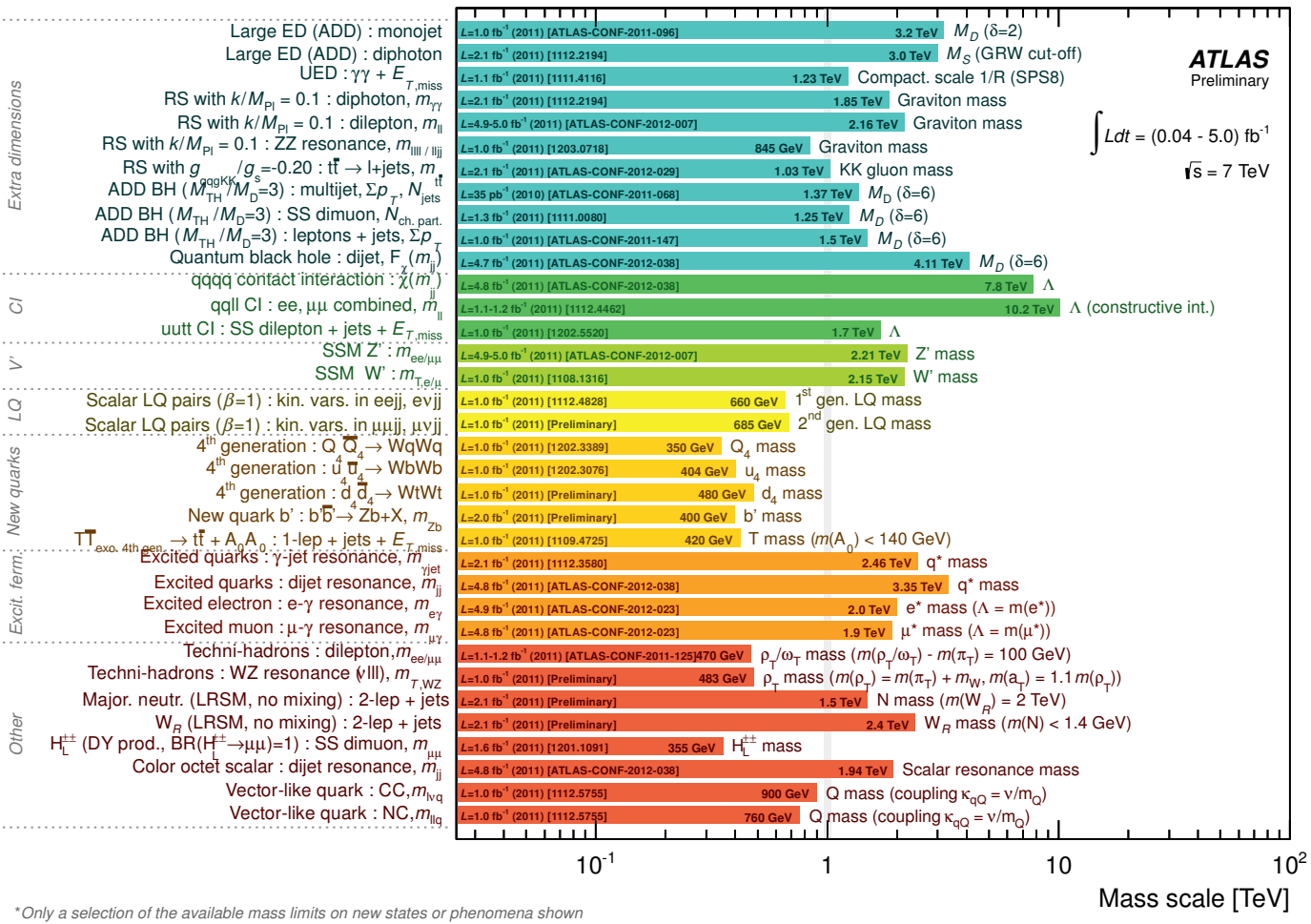

Figure 7. Mass reach of ATLAS searches for new phenomena other than Supersymmetry [2]. A representative selection of the available results at the time of the conference.

\section{References}

[1] ATLAS Collaboration, JINST 3 (2008) S08003.

[2] D. London and J. L. Rosner, Phys. Rev. D34 (1986) 1530.

[3] P. Langacker, Rev. Mod. Phys. 81 (2009) 1199-1228.

[4] J. Erler, P. Langacker, S. Munir, and E. R. Pena, JHEP 0908 (2009) 017.

[5] ATLAS Collaboration, ATLAS-CONF-2012-007, http://cdsweb.cern.ch/record/1428547. 
[6] ATLAS Collaboration, arXiv:1110.3174 [hep-ex].

[7] U. Baur, I. Hinchliffe, and D. Zeppenfeld, Int. J. Mod. Phys. A2 (1987) 1285.

[8] U. Baur, M. Spira, and P. M. Zerwas, Phys. Rev. D42 (1990) 815.

[9] ATLAS Collaboration, ATLAS-CONF-2012-038, http://cdsweb.cern.ch/record/1432206.

[10] ATLAS Collaboration, ATLAS-CONF-2012-088, http://cdsweb.cern.ch/record/1460400.

[11] G. Altarelli, B. Mele, and M. Ruiz-Altaba, Z. Phys. C45 (1989) 109.

[12] L. Randall and R. Sundrum, Phys. Rev. Lett. 83 (1999) 3370.

[13] E. Eichten and K. Lane, Phys. Lett. B669 (2008) 235.

[14] ATLAS Collaboration, Phys. Rev. D85 (2012) 112012.

[15] ATLAS Collaboration, Eur. Phys. J. C72 2056 (2012).

[16] F. Aguila, S. Bar-Shalom, A. Soni, J. Wudka, Phys. Lett. B670 (2009) 399.

[17] J.C. Pati and A. Salam, Phys. Rev. D10 (1974) 275; Erratum: Phys. Rev. D11 (1975) 703.

[18] R. Mohapatra, J.C. Pati, Phys. Rev. D11 (1975) 2558.

[19] G. Senjanovic, R.N. Mohapatra, Phys. Rev. D12 (1975) 1502.

[20] M. Cacciari and G. P. Salam, Phys. Lett. B641 (2006) 57.

[21] M. Cacciari, G. P. Salam and G. Soyez, http://fastjet.fr/.

[22] ATLAS Collaboration, https://twiki.cern.ch/twiki/bin/view/AtlasPublic/CombinedSummaryPlots. 